\title{
CD52 Positive
}

National Cancer Institute

\section{Source}

National Cancer Institute. CD52 Positive. NCI Thesaurus. Code C153221.

An indication that CD52 expression has been detected in a sample. 